\title{
Examination of Vehicle Motion Characteristics of a Maglev Train Set Using a Reduced-scale Model Experiment Apparatus
}

\author{
Hironori HOSHINO \\ Senior Researcher, \\ Erimitsu SUZUKI \\ Senior Researcher, \\ Electromagnetic Applications Laboratory, Maglev Systems Technology Division \\ Takenori YONEZU, Ph.D. \\ Researcher, \\ Ken WATANABE, Ph.D. \\ Senior Researcher, \\ Electromagnetic Applications Laboratory, Maglev Systems Technology Division
}

\begin{abstract}
This paper focuses on the vehicle dynamics of superconducting magnetically levitated (Maglev) vehicles as a train set, and reports results of computer simulation as well as results of experiments using a reduced-scale Maglev model vehicle dynamics experiment apparatus (MAGMOX). The previously constructed MAGMOX consisting of a single car was expanded to form a train set comprising several cars. The computer simulations and MAGMOX experiments produced results that demonstrated their effectiveness in reproducing testing conditions of the full-scale Maglev system that are difficult to achieve on an actual full-scale vehicle due to vehicle structure constraints and rigging space.
\end{abstract}

Keywords: superconducting Maglev, vehicle dynamics of a train set, vehicle model experiment, articulated bogie

\section{Introduction}

A superconducting magnetically levitated (Maglev) vehicle differs from a conventional wheel-on-rail vehicle with respect to vehicle dynamics in two major aspects. One difference is that the primary suspension of the Maglev train is formed by electromagnetic springs with no physical contact between the bogie and the guideway, with spring stiffness that varies with the speed of the vehicle as well as the load on the bogie; the other is that the Maglev train has articulated bogies. This paper focuses on the vehicle dynamics of Maglev vehicle as a train set, and reports results of computations and experiments using a reduced-scale Maglev model vehicle dynamics experiment apparatus (MAGMOX), both using models consisting of several car bodies and bogies.

\section{Characteristics of Maglev vehicle dynamics}

\subsection{Magnetic spring characteristics}

The primary suspension of a conventional steel-wheelon-rail railway vehicle consists of axial coil springs, while the primary suspension of a Maglev vehicle consists of magnetic springs resulting from interactions between the superconducting magnets (SCMs) onboard the vehicle and the ground coils attached to the sidewalls of the guideway. The magnetic springs suspend the vehicle such that there is no physical contact between the vehicle and the guideway, and the magnetic spring stiffness varies according to the vehicle speed and bogie load. Translational or angular displacement of a conventional spring generates a single reaction force or moment, respectively, in that same degree of freedom. In contrast, the lateral displacement of a magnetic spring, for example, not only gener- ates a lateral reaction force but also rolling and yawing reactive moments. This coupling of spring characteristics also occurs in longitudinal, vertical, and pitching movement. In the case of the Maglev vehicle, coupled lateral and rolling motion is particularly strong, and greatly influences the dynamics of the vehicle when passing through a curved guideway section at a low speed.

Furthermore, while axial dampers are installed in the primary suspension of the conventional railway vehicle to reduce vibration, no passive damping component exists in the primary suspension of the Maglev vehicle, such that the vibration acceleration peak corresponding to the magnetic spring characteristics is large. This peak exists at about 4 to $5 \mathrm{~Hz}$ for a vehicle traveling at $500 \mathrm{~km} / \mathrm{h}$, and greatly influences ride comfort in the vertical direction.

\subsection{Articulated bogie system}

The Maglev vehicle uses an articulated bogie arrangement, in which each intermediate bogie (bogies other than those at the front and rear ends of the train) is installed between two adjacent car bodies (Fig. 1). This articulated bogie arrangement increases the effectiveness of the magnetic shields, and lowers the center of gravity, improving characteristics of vehicle dynamics and reducing air resistance. The secondary suspension consists of air springs at the four corners of the bogie, a longitudinal anchor, and vertical and lateral dampers between the car body and bogie (Fig. 1, labeled in blue italic font). Because the air springs and dampers are connected to the car body directly above them, forces are transmitted between two adjacent car bodies through the articulated bogie, as well as through the coupler between the two car bodies. The transmission of forces between adjacent car bodies in the case of articulated bogies therefore differs from the case of non-articulated bogies. Therefore, examination of 


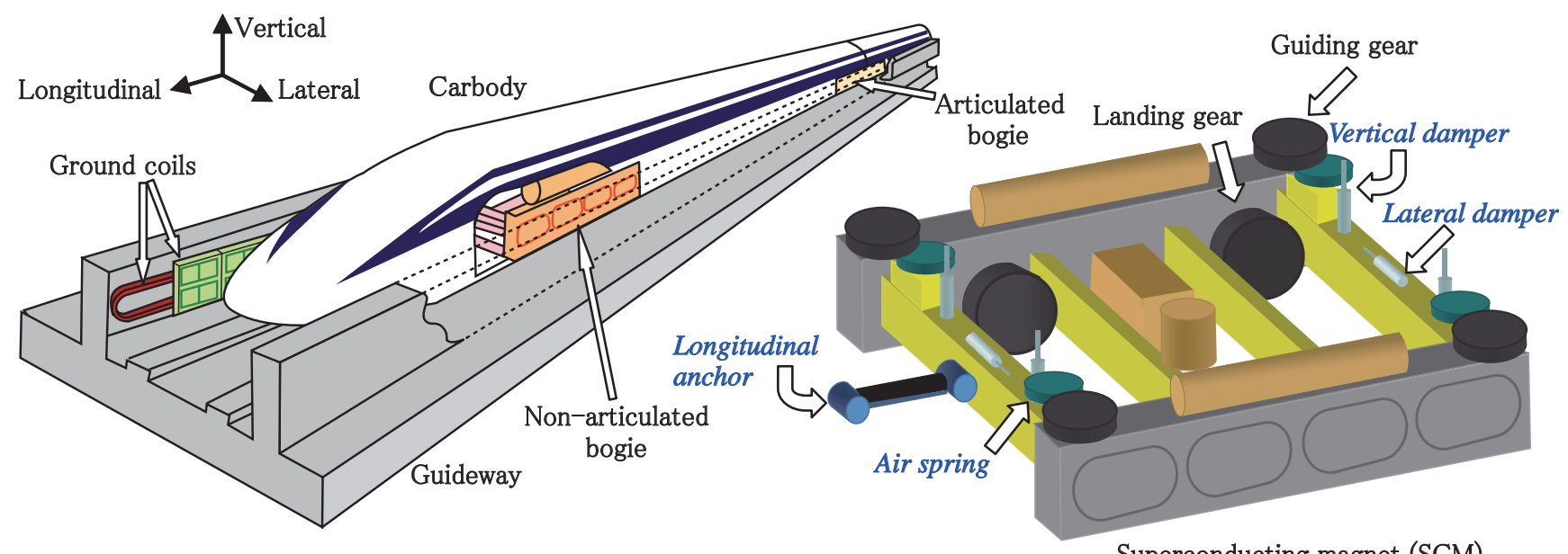

Fig. 1 Maglev vehicle and bogie

Maglev vehicle dynamics usually considers the train set as a whole rather than a single car body.

\section{Simulation of vehicle dynamics of a train set}

\subsection{Computation models}

Simulations were performed on the vehicle dynamics of a three-car Maglev train set.

The articulated bogie arrangement of the Maglev vehicle is illustrated in Fig. 2. The front car as well as the rear car of the train set has one end connected to an articulated bogie and the other end to a non-articulated bogie. Both ends of the intermediate car are attached to articulated bogies. The articulated bogie is connected to each car body at two points by way of air springs, whereas the non-articulated bogie is connected to the car body at four points. Therefore, characteristics of vehicle dynamics for the front and rear cars differ in detail from the intermediate car.

For vehicle motion in the linear domain, the computation model can be considered in the following separate forms: (1) longitudinal, vertical, and pitching motions, and (2) lateral, rolling, and yawing motions. The computation model described in this report focuses on longitudinal, vertical, and pitching motions [2].

Figure 2 shows the computation model, and Table 1 lists its main specifications. In this computation model, the car bodies and bogies were considered to be ideally
Table 1 Main specifications of the computation model [1]

\begin{tabular}{|c|c|}
\hline Parameter & Value \\
\hline Car body length (intermediate car) & $24.3[\mathrm{~m}]$ \\
\hline Bogie length & $5.4[\mathrm{~m}]$ \\
\hline Car body mass (intermediate car) & 14 [tons] \\
\hline Bogie mass & 6 [tons] \\
\hline Air spring vertical spring constant (per spring) & $0.2[\mathrm{MN} / \mathrm{m}]$ \\
\hline Damper vertical damping constant (per damper) & $5[\mathrm{kNs} / \mathrm{m}]$ \\
\hline Magnetic spring vertical spring constant (per bogie) & $5[\mathrm{MN} / \mathrm{m}]$ \\
\hline Magnetic spring pitching spring constant (per bogie) & $10[\mathrm{MN} / \mathrm{rad}]$ \\
\hline
\end{tabular}

rigid, and the number of cars was variable, with all intermediate cars having identical specifications.

\subsection{Results of computer simulations}

Examples of computer simulations of a five-car Maglev train set using the aforementioned computation model demonstrated the relationship between the height of the points at which couplers were attached to the cars and the car body pitching angular acceleration, and also examined the influence of the pitching damper between

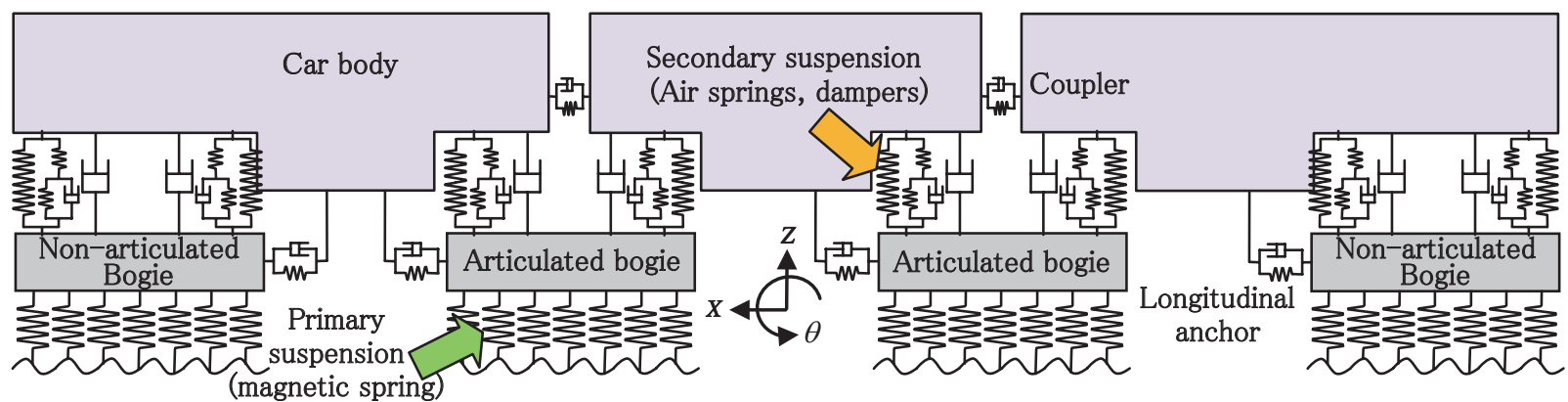

Fig. 2 Vehicle computation model for longitudinal, vertical, and pitching motions (3-car train set example) 
car bodies in improving ride comfort. All simulations described in this section investigated a Maglev vehicle traveling at a constant speed of $500 \mathrm{~km} / \mathrm{h}$.

\subsubsection{Relationship between the attachment height of the coupler and the car body pitching angular acceleration}

As in conventional railway vehicles, couplers connect adjacent car bodies of Maglev vehicles, and transmit longitudinal forces such as those of propulsion and shock absorption. In the articulated bogie arrangement of the Maglev vehicle, the floor directly above the articulated bogie at the end of the car body has a higher elevation than the floor of the passenger cabin. The coupler is therefore attached to the car bodies at a position higher than the passenger cabin floor. Using a five-car train set computation model, the attachment height of the coupler was varied to examine its relationship with the car body pitching angular acceleration.

The standard used for comparison was a coupler attached at the same height as the passenger cabin floor. This coupler attachment height was varied, and the frequency response was computed with an external disturbance consisting of sine waves of $1-\mathrm{mm}$ amplitude for frequencies in the range of 0.5 to $80 \mathrm{~Hz}$. The root-meansquare (RMS) values of the pitching angular acceleration of the third (rear) intermediate car were evaluated. An example of results is shown in Fig. 3.

The vertical distance between the standard coupler attachment height and the passenger cabin floor is indicated by d in Fig. 3. The RMS value of the pitching angular acceleration is largest when the coupler attachment height is the same as the passenger cabin floor height. The RMS value decreases as the vertical distance increases above or below this floor level.

Varying the coupler attachment height would be difficult on an actual Maglev vehicle, considering obstacles such as the gangway and machinery in the bogie. Nevertheless, the results of computations indicate that increasing the distance between the coupler attachment height and the passenger cabin floor is one possible way to reduce pitching.

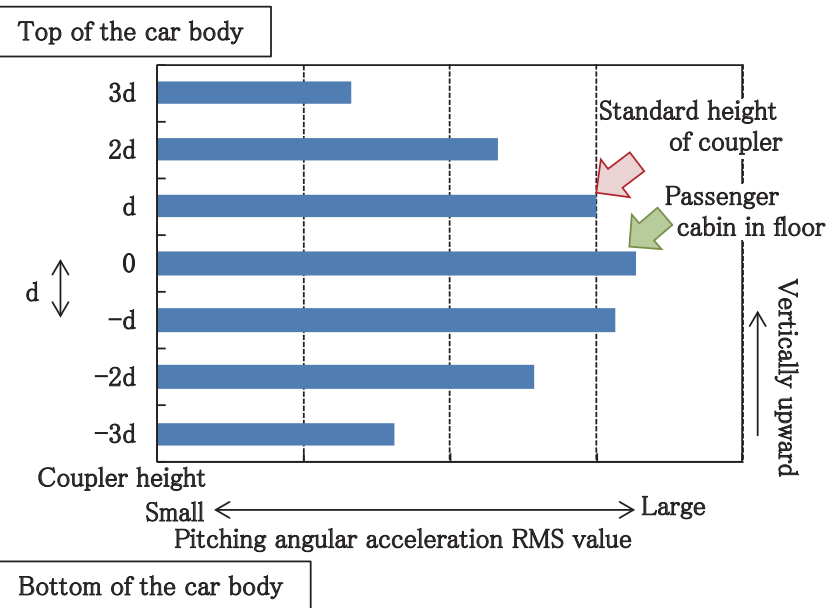

Fig. 3 Comparison of intermediate car pitching angular acceleration RMS values

\subsubsection{Effect of the pitching damper between car bodies on improving ride comfort}

A damper attached between car bodies in the conventional railway vehicle minimizes the relative angular displacement between car bodies, and contributes to the improvement of ride comfort in the vehicle traveling at high speeds. There is no damper attached between car bodies in the current Maglev vehicle due to structural constraints and rigging space in the area around the coupler attachment point. However, minimizing the relative angular displacement between car bodies when the vehicle travels at high speeds is effective in improving characteristics of vehicle dynamics of the Maglev train set. Therefore, this section focuses on longitudinal, vertical, and pitching motions, and examines the effect of hypothetically attaching a damper between car bodies to generate damping moment in proportion to the relative angular velocity between the car bodies (pitching damper). An example of a pitching damper is illustrated in Fig. 4.

Simulations were performed using a computation model of a five-car train set and external disturbance generated by simulated vertical guideway irregularities with the power spectral density (PSD) characteristics shown in Fig. 5. The vertical acceleration PSD of the end of the car body was derived from the time-domain response (Fig. 6). The effect of the pitching damper was evaluated by the ride comfort level derived from the vertical accel-

\section{Pitching damper (rotary damper)} generates damping moment in proportion to angular velocity

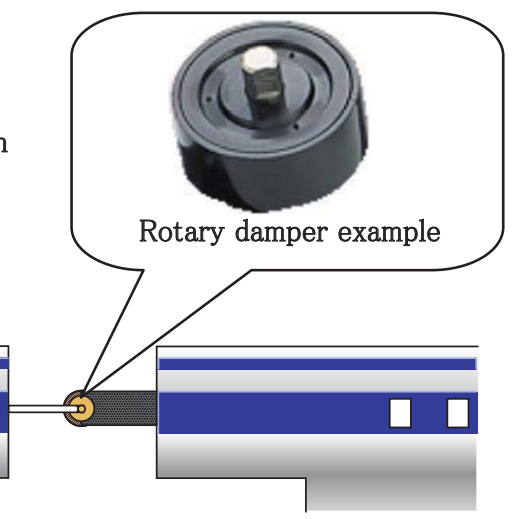

Fig. 4 Example of a pitching damper

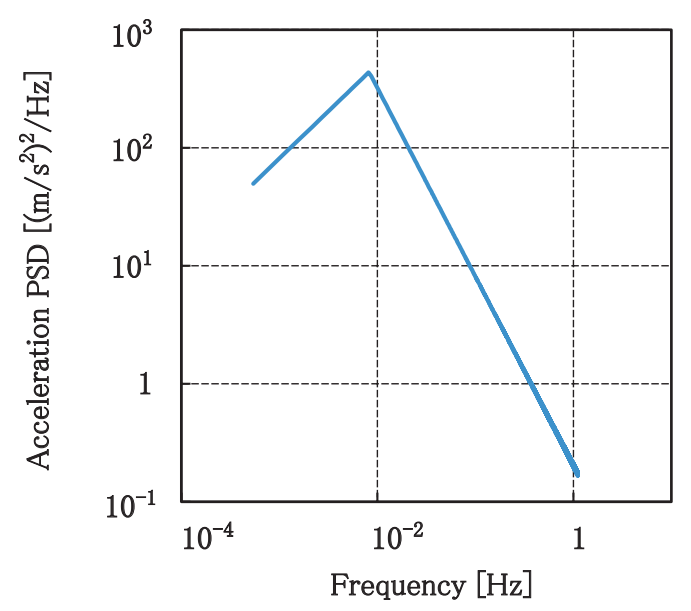

Fig. 5 Simulated vertical guideway irregularity PSD 


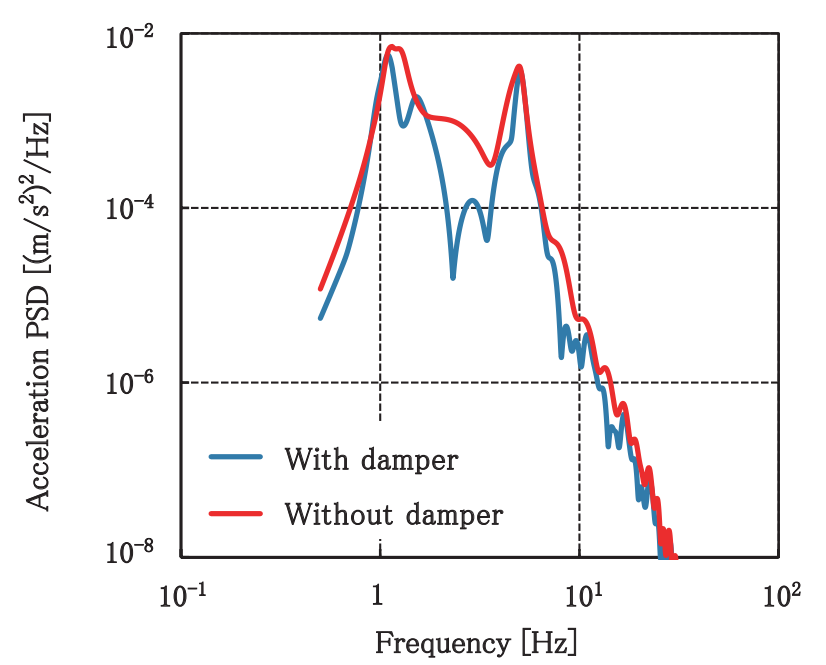

Fig. 6 Example of a comparison of car body end vertical acceleration PSD (Pitching damper damping coefficient $\left.1.0 \times 10^{2}[\mathrm{MN} \cdot \mathrm{m} \cdot \mathrm{s} / \mathrm{rad}]\right)$

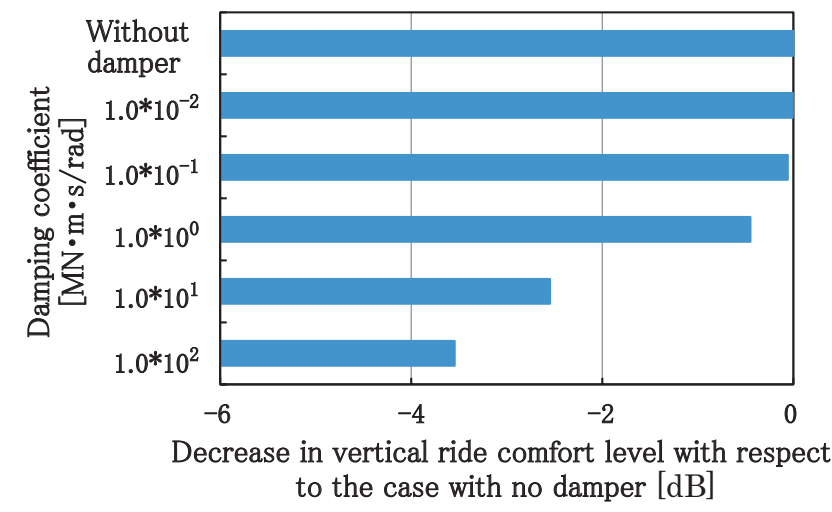

Fig. 7 Effect of the pitching damper attached between car bodies

eration of the end of the third (rear) car body.

Figure 7 shows an example of results examining the effect of the damping coefficient of the pitching damper on the vertical ride comfort level. This same figure confirms that attaching this pitching damper can improve the vertical ride comfort level by a maximum of $4 \mathrm{~dB}$ with respect to a vehicle without the pitching damper.

\section{Reproduction of motion of a Maglev train set using MAGMOX}

\subsection{Structure of MAGMOX}

\subsubsection{Overview of the experiment apparatus}

MAGMOX reproduces motion of the traveling Maglev vehicle, and was constructed to confirm results of computer simulations and the effects of vibration control [3], [4]. The initial setup consisted of one car body and two bogies [3], but additional components were built to expand the setup to two and a half car bodies and three bogies, to test a train set (Fig. 8). The main specifications of each bogie and car body are the same as those used in Reference [3]. Because the MAGMOX experiments were conducted in a standard gravitational field, the laws of similitude were set such that the magnitudes of acceleration in the reducedscale model matched those of the full-scale vehicle. Consequently, the vibration frequency in MAGMOX was about $3.4(\sqrt{12})$ times that of the full-scale vehicle, such that, for example, the acceleration peak at about $5 \mathrm{~Hz}$ corresponding to the primary suspension was equivalent to about 17 $\mathrm{Hz}$ in the 1/12-scale model.

Modifications to the secondary suspension improved the performance of MAGMOX in reproducing vehicle motion [5]. Figure 9 shows the secondary suspension unit after modifications.

A hydraulically-powered motion base (Stewart-type parallel link mechanism) was used to reproduce motions of the bogie, and was able to reproduce the characteristics of magnetic springs that would otherwise be difficult to reproduce using only mechanical springs. Furthermore, load cells were attached to the bottom of the secondary suspension unit to measure the forces interacting between the car body and bogie. Using these force measurements as feedback in the bogie motion control device, MAGMOX was able to reproduce coupled motions of the car body and bogie.

By reproducing these coupled motions, changes in the forces that interact between the car body and bogie can be incorporated into the bogie motion in real time. Therefore,

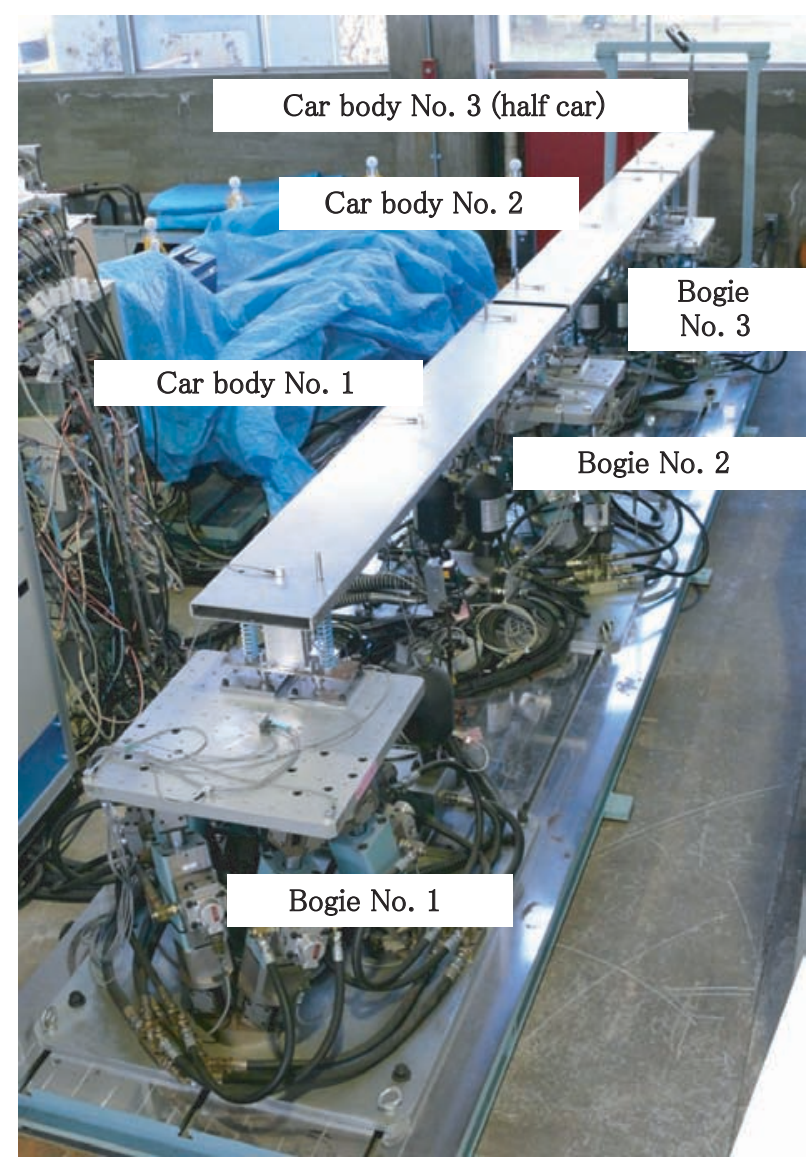

Fig. 8 MAGMOX apparatus expanded to test a 2.5-car train set 


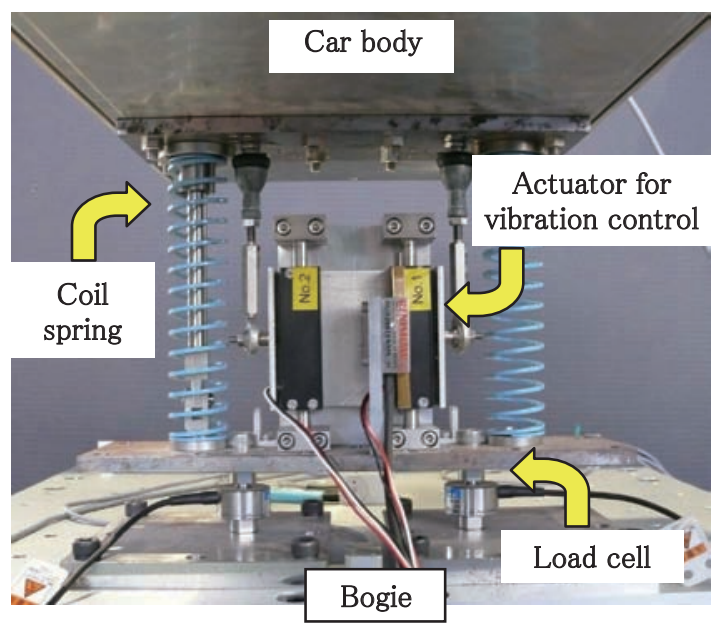

Fig. 9 Secondary suspension unit

vibration control by active suspension can be applied to the secondary suspension, using an electromagnetic actuator (shaft motor) attached in parallel with the coil spring.

Because of restrictions such as the limited space in and around the secondary suspension unit, the experiments of this phase using the 1/12-scale car body were performed with the bogie limited to only the vertical motion, and the car body to only vertical and pitching motions. Simulations were performed on the vehicle dynamics of a three-car Maglev train set.

\subsubsection{Expansion of the experiment apparatus to test a train set}

As mentioned above, the initial MAGMOX setup consisted of one car body and two bogies, but additional car bodies and a bogie were built to expand the setup to reproduce motions of a train set. Two types of car bodies were newly built, one to reproduce motions of the front car with an articulated bogie on one end and a non-articulated bogie on the other end, and the other type with the same specifications as the already-existing car body, to reproduce motions of the intermediate car with both ends attached to articulated bogies. One new bogie was built and installed on MAGMOX alongside the two already-existing bogies, but no more could be added to the setup, because of space constraints and the maximum output limit of the hydraulic power source. Consequently, the final new car body was built with the length being half that of the intermediate car. One end of this half-car was attached to an articulated bogie, and the other end was suspended by a wire.

Ball joints were used on both ends of the coupler between car bodies to avoid the effects of rotational motion, just as in the full-scale vehicle. In order to examine how the characteristics of vehicle dynamics are influenced by changing the position in which the coupler is attached to the car bodies, four different attachment positions numbered (1) to (4) were considered, as shown in Fig. 10. Each position results in a different height of the axis of rotation for the pitching motion of the car bodies. The setup without any coupler between the car bodies was assigned the case number (5). Figure 11 shows an example of a coupler attached in position (4).

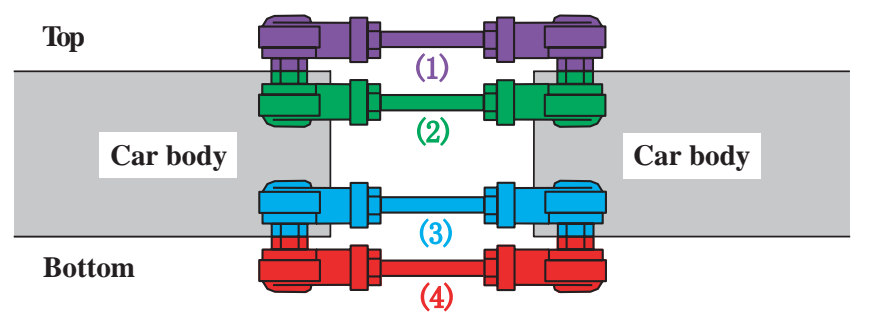

Fig. 10 Coupler attachment positions

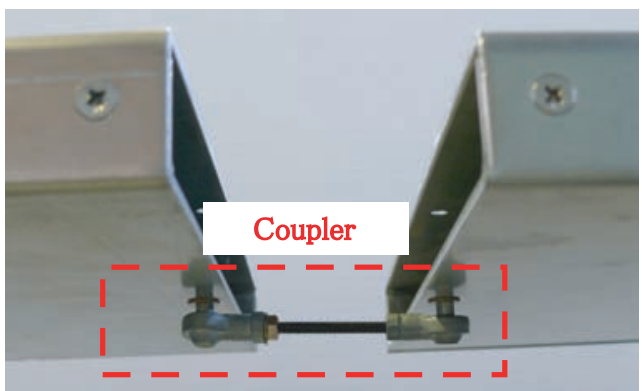

Fig. 11 Coupler attached in position (4)

\subsection{Results of experiments}

MAGMOX experiments reproduced vertical and pitching motions of a train set for the passive case without any vibration control of the secondary suspension. The results of experiments were compared with those of simulations to confirm the reproduction of the vehicle dynamics of the train set.

The MAGMOX bogie (motion base) was modeled by a 1-DOF (one degree of freedom) system, using the forces of interaction in the secondary suspension and guideway vertical irregularities as external disturbance input. The vertical irregularities of the guideway were the same as those used in Reference [3]. The bogie displacement was computed by solving the dynamic equations of this 1-DOF system in real time, and the bogie motion was controlled using vertical displacement of the bogie as feedback.

MAGMOX experiments were conducted for the five cases of coupler attachment positions described above, and the accelerations of the car bodies and bogies were measured when vibrating in response to the external disturbance input.

Figure 12 shows an example of time-domain plots of the vertical acceleration of the front and rear ends of car body No. 1, for coupler attachment position (4). Figure 13 shows PSD plots of this vertical acceleration data. The results show an acceleration peak at around $17 \mathrm{~Hz}$ corresponding to the primary suspension, which agrees with the results for the setup using one car body and two bogies [5]. The peak in the range of 5 to $9 \mathrm{~Hz}$ can be considered as a combination of the peak corresponding to the secondary suspension and the peak corresponding to the car body pitching motion.

Next, in order to confirm the characteristics of the vehicle dynamics as a train set, experiments were conducted with and without the coupler attached between car bodies, to examine the influence of the coupler attachment position on the car body pitching angular acceleration. 


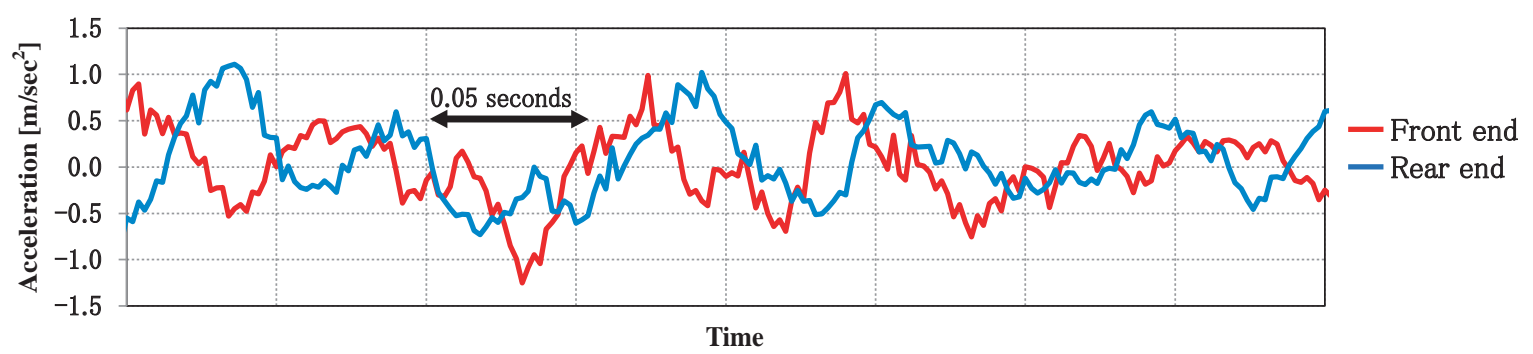

Fig. 12 Car body No. 1 vertical acceleration time-domain plot, for coupler attachment position (4)

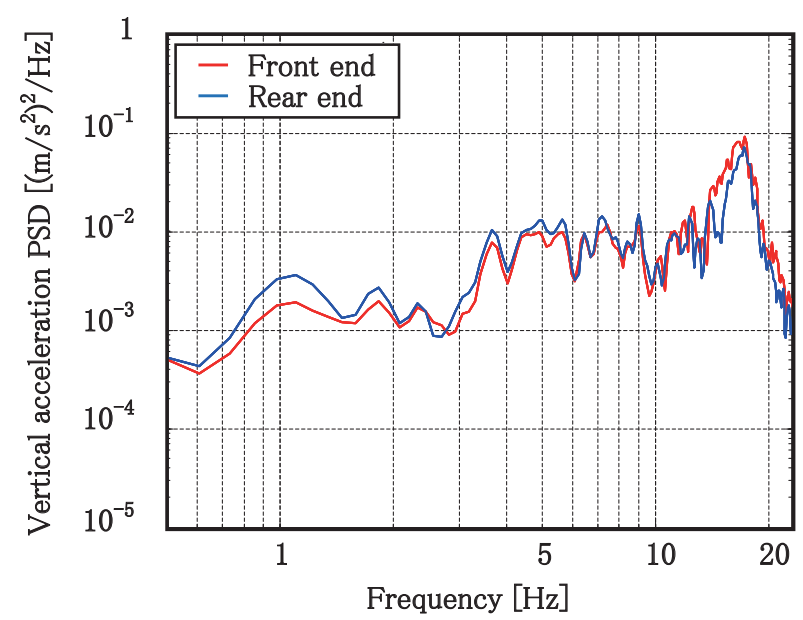

Fig. 13 Example of car body No. 1 vertical acceleration PSD, for coupler attachment position(4)

Figure 14 shows the pitching angular acceleration PSD plots for the cases (4) and (5). For the case (5) without any coupler between car bodies, an acceleration peak appears at around $8 \mathrm{~Hz}$ corresponding to the car body pitching motion. However, when the coupler is attached at position (4), the car body pitching angular acceleration peak is reduced to the extent that the peak is hardly noticeable, confirming that pitching is reduced when the car body is constrained by the coupler.

Experiments were also conducted to examine how changing the coupler attachment position influenced the aforementioned angular acceleration peak at around $8 \mathrm{~Hz}$ corresponding to the car body pitching motion. The five cases of coupler attachment positions were compared in terms of the RMS value of the car body No. 1 pitching angular acceleration, which was derived by integrating the angular acceleration PSD for the frequencies around the peak mentioned above $(8.6$ to $9.9 \mathrm{~Hz})$. An example of results is shown in Fig. 15, with the RMS values normalized with respect to the case (5) without any coupler.

The same figure also shows that the RMS value of the car pitching angular acceleration for all four coupler attachment positions is smaller than in the case without any coupler. Furthermore, the RMS value decreases as the coupler attachment position moves farther vertically from the center of the car body. The trend shown in this result is consistent with that of the computer simulation result described in the previous chapter.

Because the rigging space is limited, the coupler used in MAGMOX is not designed to accurately model the parameters of an actual coupler of a full-scale vehicle. Con-

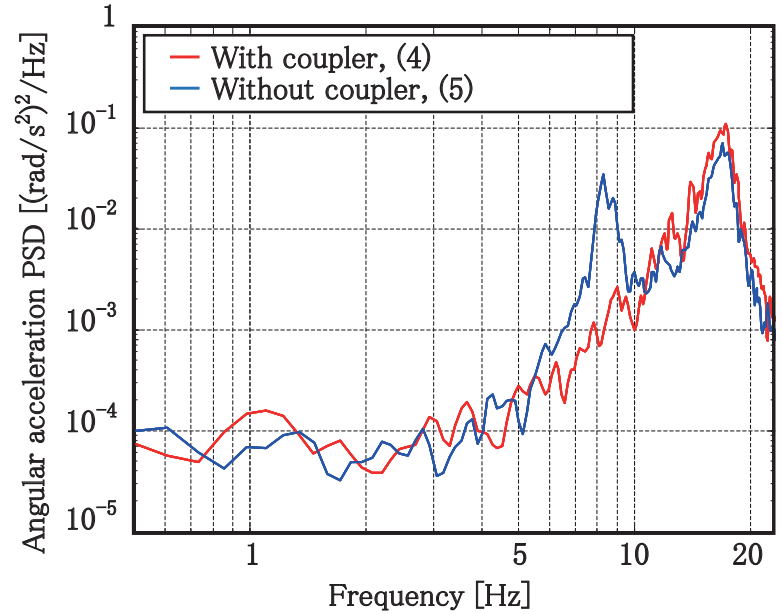

Fig. 14 Example of a comparison of car body No. 1 pitching angular acceleration PSD

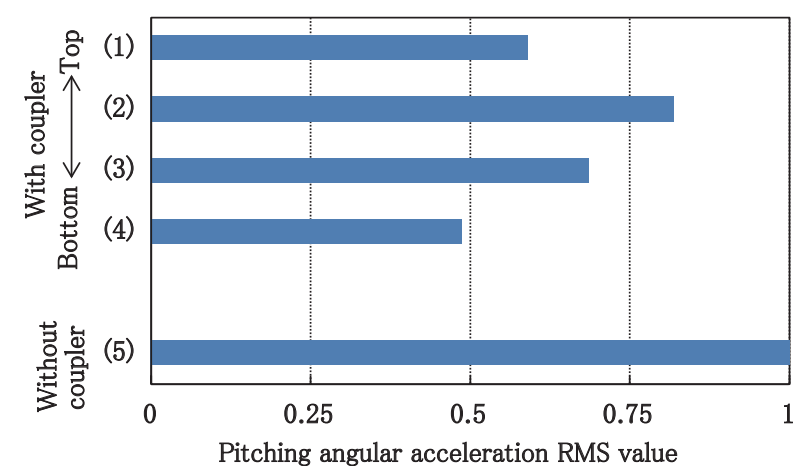

Fig. 15 Example of a comparison of car body No. 1 pitching angular acceleration RMS values

sequently, it is difficult to quantitatively match the results of MAGMOX experiments with those of the computer simulations that model the full-scale vehicle. However, it is possible to qualitatively compare trends such as the one mentioned in the previous paragraph.

\section{Conclusions}

This study focused on vehicle dynamics of the Maglev system as a train set, and the results of examining characteristics of vertical and pitching motions of the car body led to the following conclusions:

- Simulations of vehicle dynamics demonstrated that attaching anti-pitching dampers between car bodies 
is effective in improving ride comfort.

- Computer simulations and MAGMOX experiments produced results that showed how the height at which the coupler is attached between car bodies influences the car pitching motion. And, the expansion of the experiment apparatus to test a train set demonstrated the effectiveness of MAGMOX in examining the characteristics of vehicle dynamics of the Maglev train set.

The two examples of studies mentioned above are both difficult to perform on an actual full-scale vehicle, due to limitations of the vehicle structure and rigging space. The computer simulations and MAGMOX experiments produced results that demonstrated their effectiveness in reproducing testing conditions of the actual Maglev system.

\section{Future work}

Future work under consideration includes using MAGMOX to examine the effectiveness of applying various control laws to reduce vibration in the Maglev train set [6], and using a larger-scale car body model in the experiments to expand the setup to include examination of longitudinal, lateral, and rolling motions.

This work has been financially supported in part by the Ministry of Land, Infrastructure, Transport and Tourism of the Government of Japan.

\section{References}

[1] Yoshioka, H. and Watanabe, K., "Dynamic Characteristics of a Sidewall Magnetically Levitated Vehicle," RTRI Report, Vol. 8, No. 10, pp. 29-34, 1994, (in Japanese).

[2] Yoshioka, H., "Dynamic Model of Maglev Vehicle," RTRI Report, Vol. 2, No. 6, pp. 17-22, 1988, (in Japanese).

[3] Suzuki, E., Watanabe, E., and Hoshino, H.: Examination of Fundamental Characteristics of Vehicle Dynamics Using a Maglev Vehicle Model Experiment Apparatus, RTRI Report, Vol. 22, No. 11, pp. 5-10, 2008, (in Japanese).

[4] Watanabe, K., Suzuki, E., Yonezu, T., and Hoshino, H., "Simulation of Vehicle Dynamics Using a Maglev Vehicle Model Experiment Apparatus," Mechanical Engineering Congress, of the Japan Society of Mechanical Engineers (MECJ-09), 2009, (in Japanese).

[5] Suzuki, E., Watanabe, K., Hoshino, H., Yonezu, T. and Nagai, M., "A Study of Maglev Vehicle Dynamics Using a Reduced-Scale Vehicle Model Experiment Apparatus," Journal of Mechanical Systems for Transportation and Logistics, of the Japan Society of Mechanical Engineers (JMTL-JSME), Vol. 3, No. 1, pp. 196-205, 2010.

[6] Suzuki, E., Watanabe, E., Hoshino, H. and Nagai, M., "An Examination of Active Electromagnetic and Mechanical Suspension Control of Superconducting Maglev Vehicles," Journal of the Japan Society of Applied Electromagnetics and Mechanics (JSAEM), Vol. 17, No. 2, pp. 324-330, 2009. 